\title{
Velocity and temperature derivatives in high-Reynolds- number turbulent flows in the atmospheric surface layer. Part 2. Accelerations and related matters
}

\section{Journal Article}

\section{Author(s):}

Gulitski, G.; Kholmyansky, M.; Kinzelbach, W.; Lüthi, B.; Tsinober, A.; Yorish, S.

Publication date:

2007

Permanent link:

https://doi.org/10.3929/ethz-b-000004734

Rights / license:

In Copyright - Non-Commercial Use Permitted

Originally published in:

Journal of Fluid Mechanics 589, https://doi.org/10.1017/S0022112007007501 


\title{
Velocity and temperature derivatives in high-Reynolds-number turbulent flows in the atmospheric surface layer. Part 2. Accelerations and related matters
}

\author{
G. GULITSKI I M. KHOLMYANSKY I, W. KINZELBACH${ }^{2}$, \\ B. LÜTHI ${ }^{2}$, A. TSINOBER ${ }^{1}$ AND S. YORISH ${ }^{1}$ \\ ${ }^{1}$ Faculty of Engineering, Tel Aviv University, Tel Aviv 69978, Israel \\ ${ }^{2}$ Institute of Environmental Engineering, ETH Zürich, \\ CH-8093 Zürich, Switzerland
}

(Received 11 January 2006 and in revised form 19 May 2007)

We report the first results of an experiment, in which explicit information on all velocity derivatives (the nine spatial derivatives, $\partial u_{i} / \partial x_{j}$, and the three temporal derivatives, $\partial u_{i} / \partial t$ ) along with the three components of velocity fluctuations at a Reynolds number as high as $R e_{\lambda} \sim 10^{4}$ is obtained. No use of the Taylor hypothesis was made, and this allowed us to obtain a variety of results concerning acceleration and its different Eulerian components along with vorticity, strain and other smallscale quantities. The field experiments were performed at five heights between 0.8 and $10 \mathrm{~m}$ above the ground.

The report consists of three parts. Part 1 is devoted to the description of facilities, methods and some general results. Part 2 concerns accelerations and related matters. Part 3 is devoted to the issues concerning temperature with the emphasis on joint statistics of temperature and velocity derivatives.

\section{Introductory notes}

As the material derivative of the velocity vector, the fluid particle acceleration field in turbulent flow is among the natural physical parameters of special interest in turbulence research for a variety of reasons. Problems in which fluid particle acceleration plays a key role range from studies of basic issues such as finescale intermittency, production of Reynolds stresses, the so-called random Taylor hypothesis and two-phase turbulent flows to applications in turbulent mixing and transport, cloud physics and influence of turbulence on the behaviour of insects. In particular, Lagrangian acceleration statistics are at the core of the kinematic theory and modelling of turbulent dispersion, mixing, particulate transport and combustion. Finally, the acceleration gradient tensor is known to govern the topology of quasi-geostrophic stirring (particle dispersion and tracer gradient evolution) and transport properties in nearly two-dimensional and geostrophic turbulence (Yeung \& Pope 1989; Borgas \& Sawford 1991; Bernard, Thomas \& Handler 1993; Hua \& Klein 1998; La Porta et al. 2001; Tsinober, Vedula \& Yeung 2001; Pope 2002; Shaw 2003).

Hence, it is not surprising that there is a huge interest in a variety of issues associated with fluid particle accelerations in turbulent flows (Hill \& Wilczak 1995; 

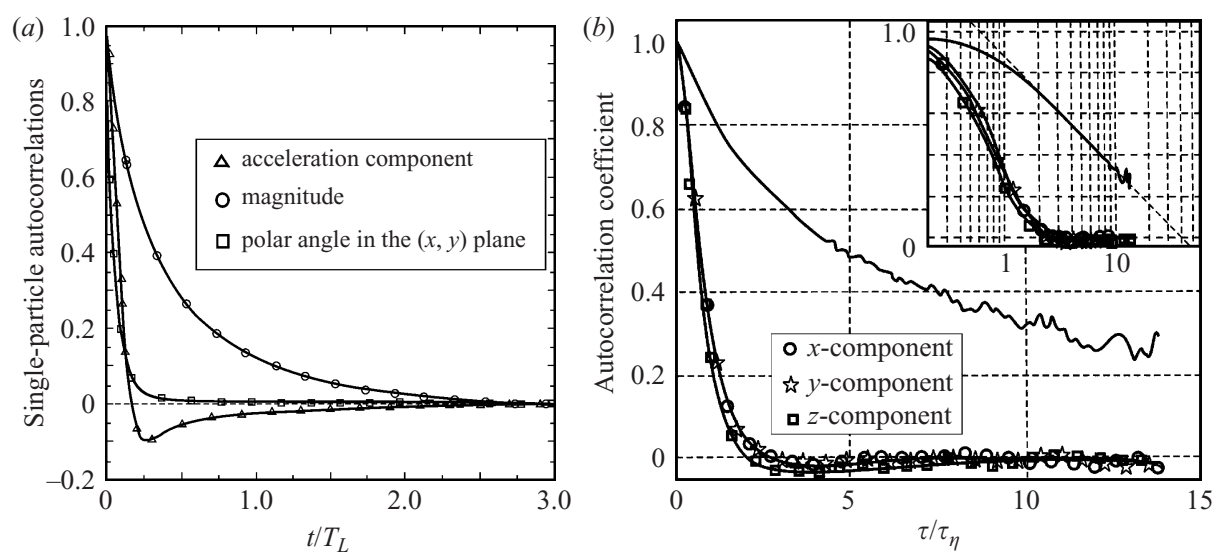

FIGURE 1. Autocorrelation functions of the acceleration, $\boldsymbol{a}$, and its components. (a) From Yeung (1997) with time scaled by the Lagrangian velocity integral time scale at $R e_{\lambda}=140$. (b) From Mordant et al. (2004b) with time scaled by the Kolmogorov time $\tau_{\eta}=\sqrt{\nu / \varepsilon}$, the line without symbols is for the acceleration magnitude, inset: semilogarithmic plot.

Hill \& Thoroddsen 1997; Yeung 1997; Voth et al. 1998, 2002; Mann, Ott \& Andersen 1999; Vedula \& Yeung 1999; Ott \& Mann 2000; Pinsky, Khain \& Tsinober 2000; Christensen \& Adrian 2001, 2002a,b; Kholmyansky, Tsinober \& Yorish 2001b; La Porta et al. 2001; Lüthi et al. 2001; Shaw \& Oncley 2001; Tsinober et al. 2001; Hill 2002; Mordant et al. 2003, 2004a-c; Sawford et al. 2003; Aringazin \& Mazhitov 2004; Biferale et al. 2004; Gylfason, Ayyalasomayajula \& Warhaft 2004; Lee, Yeo \& Choi 2004; Yeung \& Borgas 2004; Crawford, Mordant \& Bodenschatz 2005; Reynolds et al. 2005; Chen, Goto \& Vassilicos 2006 and references therein).

The study of the basic properties of Lagrangian accelerations was started using direct numerical simulations by Yeung \& Pope (1989) and continued by Yeung (1997); Vedula \& Yeung (1999) and Tsinober et al. (2001). Because of extreme difficulties, the direct measurement of the acceleration of a fluid particle has only recently been achieved in laboratory flows by three-dimensional particle tracking (3DPT) (see Mann et al. 1999; Ott \& Mann 2000; La Porta et al. 2001; Lüthi et al. 2001; Mordant et al. 2003, 2004b; Sawford et al. 2003; Crawford et al. 2005; Reynolds et al. 2005), with especially high-precision experiments performed by E. Bodenschatz and colleagues. These experiments were made in a water flow configuration known as the 'French washing machine' in which the flow is produced by two counter-rotating disks (as in the classical von Kármán flow) and is believed to be highly anisotropic. Among the findings in the direct determination of particle acceleration (both via DNS and 3DPT) is the persistence of the magnitude of accelerations along the fluid particle trajectory as contrasted with the variability of its direction. The former has a correlation time of the order of integral Lagrangian time, whereas the latter has a correlation time of the Kolmogorov time only (see figure 1).

In the Eulerian setting, a successful attempt to measure accelerations was made in a turbulent channel flow by particle-image accelerometry (Christensen \& Adrian $2001,2002 a, b$ ) which is an extension of the particle image velocimetry at moderate Reynolds number using air as a working fluid. 


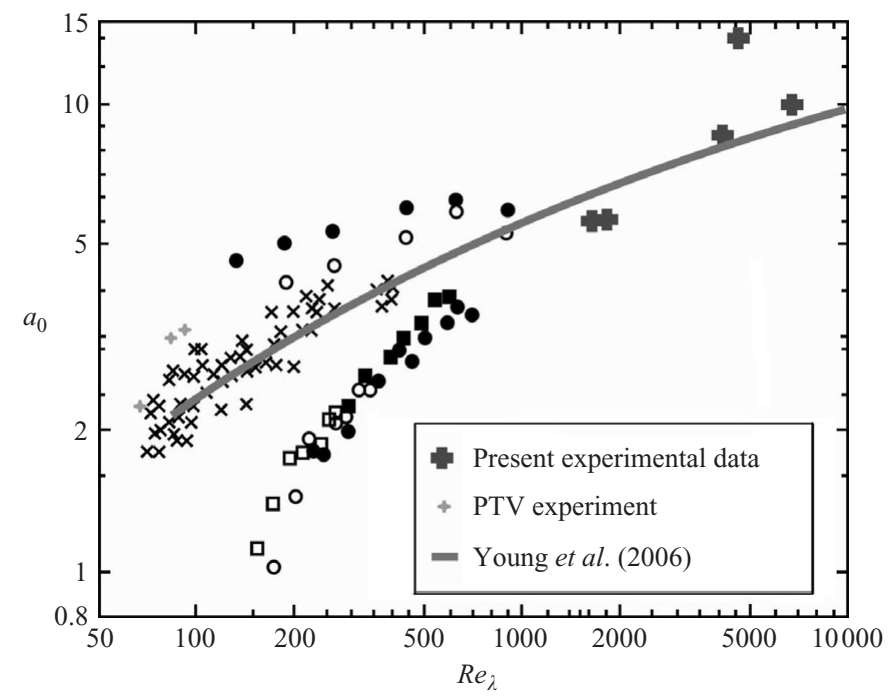

FIGURE 2. Normalized acceleration variance, $a_{0}=(1 / 3)\left\langle a_{k} a_{k}\right\rangle \epsilon^{-3 / 2} v^{1 / 2}, v s$. $R e_{\lambda}$ from Gylfason et al. (2004). Points are from the present experimental data and from the PTV experiments (Lüthi et al. 2005). The line is the fit from Yeung et al. (2006): $a_{0}=1.3 / R e_{\lambda}^{0.22}+$ $0.88 R e_{\lambda}^{0.06} \ln \left(R e_{\lambda} / 20\right)$.

Until recently, it was believed to be impossible to use hot-wire anemometry to measure acceleration:

Even in multipoint probe measurements of velocity gradients (e.g. Vukoslavcevic, Wallace, and Balint (1991); Tsinober, Kit, and Dracos (1992)), Taylor's hypothesis is invoked to estimate derivatives along the mean streamwise direction. (Dahm \& Southerland 1997).

In order to determine $\boldsymbol{a}$ from Eulerian measurements, it would be necessary to know $\partial \boldsymbol{u} / \partial t$ as well as $\boldsymbol{u}$ and $\boldsymbol{\nabla u}$ at a point in space (which is possible in DNS but not in experiments). (Voth et al. 2002)

The only results achievable so far relate to indirect evaluation of acceleration variance, $\left\langle a^{2}\right\rangle$, using the methods developed by Hill and Wilczak assuming local isotropy (Hill \& Wilczak 1995; Hill \& Thoroddsen 1997) and used by Gylfason et al. (2004). Figure 2 summaries various results on the acceleration variance normalized by Kolmogorov scaling, $a_{0}=\left\langle a_{k} a_{k}\right\rangle \epsilon^{-3 / 2} v^{1 / 2} / 3$. Also shown are additional results from recent particle tracking data, recorded with the 3D-PTV system of Risø National Laboratory (Ott \& Mann 2000; Jørgensen et al. 2005; Mann et al. 2005) and processed using the method of Lüthi, Tsinober \& Kinzelbach (2005) and from some of our recent field experiments described below. Though all the results are of the same order, the scatter is not small.

However, it appears that it is possible to measure instantaneous accelerations in an Eulerian setting using hot-wire anemometry. We made such a successful attempt (Kholmyansky, Tsinober \& Yorish 2001a; Galanti et al. 2003) through an extension of the multi-hot-wire technique developed by Tsinober and colleagues (Tsinober et al. 1992, 1997; Kholmyansky \& Tsinober 2000; Kholmyansky et al. 2000, 2001a, b; Busen et al. 2001; Galanti et al. 2003, 2004). The new version of this technique 

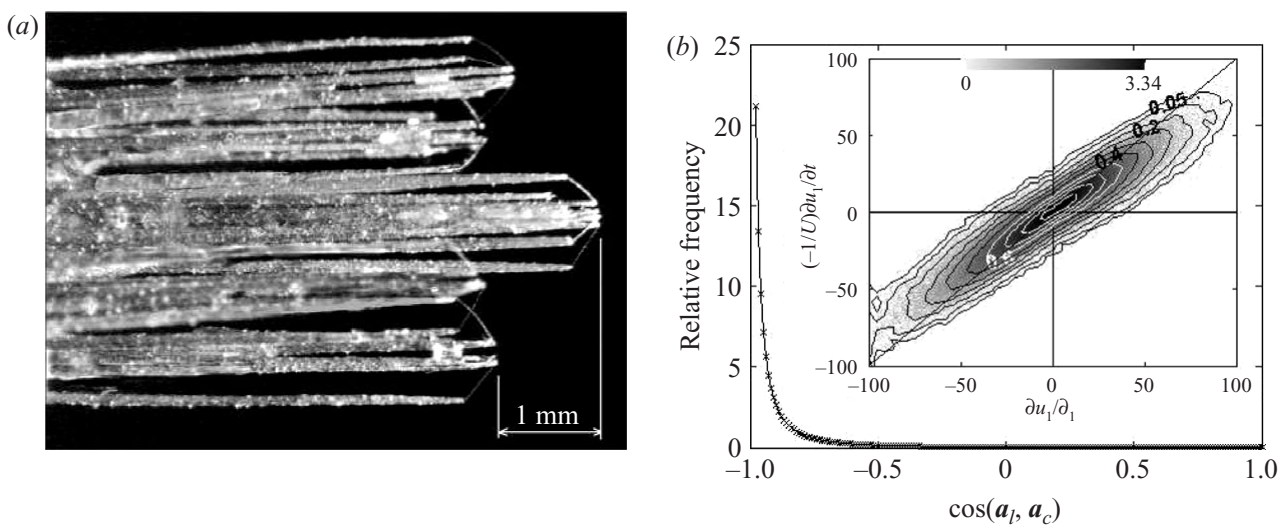

FIGURE 3. (a) Tip of the NTH-probe; (b) PDF of the cosine between the local, $\boldsymbol{a}_{l}$, and convective, $\boldsymbol{a}_{c}$, acceleration components. Inset: joint PDF of the true longitudinal derivative, $\partial u_{1} / \partial x_{1}$, and its estimate using the Taylor hypothesis, $(-1 / U) \partial u_{1} / \partial t$. Correlation coefficient $=0.930$.

allowed us to estimate the spatial derivatives of all three velocity components in the streamwise direction independently of the time derivative, i.e. without invoking the Taylor hypothesis.

This was achieved by constructing a five-array probe (see figure $3 a$ ) with the central array shifted out in the streamwise direction: the so-called NTH-probe ("non-Taylor hypothesis'). Figure 3(b) shows one of the earlier results obtained with such a probe in an atmospheric surface layer (Kholmyansky et al. 2001b). It is related to the random Taylor hypothesis (Tennekes 1975) and demonstrates strong anti-alignment between the Eulerian components, $\boldsymbol{a}_{l}=\partial \boldsymbol{u} / \partial t$ (local) and $\boldsymbol{a}_{c}=u_{k}\left(\partial \boldsymbol{u} / \partial x_{k}\right)=(\boldsymbol{u} \cdot \nabla) \boldsymbol{u}$ (convective), of the fluid particle acceleration, $\boldsymbol{a}=\boldsymbol{a}_{l}+\boldsymbol{a}_{c}$. This effect was also observed in DNS at relatively low Reynolds numbers (Tsinober et al. 2001). The strong antialignment of $\boldsymbol{a}_{l}$ and $\boldsymbol{a}_{c}$ is a manifestation of their strong mutual cancellation, so that $\boldsymbol{a}$ appears to be much smaller than both $\boldsymbol{a}_{l}$ and $\boldsymbol{a}_{c}$, which represents the main difficulty in reliable determination, e.g. of the variance of $\boldsymbol{a}$. Thus, we have already shown (Kholmyansky et al. 2001b; Galanti et al. 2003) that it is possible to use hot-wire techniques for studying the field of fluid particle acceleration in an Eulerian setting. (The reason that the slope in figure $3(b)$ is not along the bisector is a combination of errors in the estimation of $\mathrm{d} x_{1}$ (geometry and effective 'centre of mass') and mean velocity (calibration errors; it is well known that the mean velocity is difficult to obtain from the hot-wire measurements). At the present level of precision we relate the thickness of the ellipse to the calibration errors and do not draw conclusions about the Taylor hypothesis.)

The main reason why we are using an 'old-fashioned technique' such as hot-wire anemometry is that it gives us the possibility of measuring all three components of the velocity fluctuations vector, $u_{i}$, and all nine components of the spatial velocity gradients tensor, $\partial u_{i} / \partial x_{j}$, and its time derivatives, $\partial u_{i} / \partial t$. So far, all 'modern' techniques such as particle image velocimetry (PIV), particle tracking velocimetry (PTV), holography and others are unable to cope with this problem either in resolution or in the essence, i.e. obtaining the full set (total 15) of velocity derivatives both in space and time at Reynolds numbers $\sim 10^{4}$. 


\subsection{Main goals and tasks}

The preliminary results mentioned above comprise the basis for setting the main theme and objectives of the present work.

For convenience we, first, recall here some of the notations.

The acceleration is $\boldsymbol{a}=\boldsymbol{a}_{l}+\boldsymbol{a}_{c}$, where $\boldsymbol{a}_{c}=(\boldsymbol{u} \cdot \nabla) \boldsymbol{u}$ is the convective acceleration and $\boldsymbol{a}_{l}=\partial \boldsymbol{u} / \partial t$ is the local acceleration in the frame attached to the ground, $\boldsymbol{a}_{L}=\boldsymbol{\omega} \times \mathbf{u}$ is the Lamb vector, $\boldsymbol{a}_{B}=\nabla u^{2} / 2$ is the Bernoulli component of acceleration, $\boldsymbol{a}_{\|}=(\boldsymbol{a} \cdot \widehat{\boldsymbol{u}}) \widehat{\boldsymbol{u}}$ is the acceleration component parallel to the velocity vector, where $\widehat{\boldsymbol{u}}=\boldsymbol{u} / \boldsymbol{u}$ is the unit vector along the velocity vector, and $\boldsymbol{a}_{\perp}=\boldsymbol{a}-\boldsymbol{a}_{\|}$is the acceleration component normal to the velocity vector.

In addition, we consider $\boldsymbol{a}_{c}^{\prime}=\{(\boldsymbol{u}-\boldsymbol{U}) \cdot \nabla\} \boldsymbol{u}$ and $\boldsymbol{a}_{l}^{\prime}=\partial \boldsymbol{u} / \partial t+(\boldsymbol{U} \cdot \nabla) \boldsymbol{u}$ which are the convective and local components of acceleration in a frame moving with mean velocity, $\boldsymbol{U}$, and are different from $\boldsymbol{a}_{l}$ and $\boldsymbol{a}_{c}$, i.e. although the particle acceleration is a Galilean invariant quantity, its Eulerian components are not. In a frame moving with constant (mean) velocity, $\boldsymbol{U}$, the velocity time derivative (i.e. local acceleration, $\boldsymbol{a}_{l}$, in a moving frame which we denote as $\left.\boldsymbol{a}_{l}^{\prime}\right)$ is

$$
\lim _{\tau \rightarrow 0} \frac{\boldsymbol{u}(\boldsymbol{x}+\boldsymbol{U} \tau, t+\tau)-\boldsymbol{u}(\boldsymbol{x}, t)}{\tau}=\frac{\partial \boldsymbol{u}}{\partial t}+(\boldsymbol{U} \cdot \nabla) \boldsymbol{u} .
$$

The space derivative, e.g. $\partial / \partial x_{1}$, is

$$
\lim _{\xi \rightarrow 0} \frac{\boldsymbol{u}(\boldsymbol{x}+\xi \boldsymbol{i})-\boldsymbol{u}(\boldsymbol{x})}{\xi}=\frac{\partial \boldsymbol{u}}{\partial x_{1}},
$$

but the instantaneous velocity is $\boldsymbol{u}-\boldsymbol{U}$. Therefore, the convective derivative (i.e. the convective acceleration, $\boldsymbol{a}_{c}$, which we denote as $\left.\boldsymbol{a}_{c}^{\prime}\right)$ becomes $\{(\boldsymbol{u}-\boldsymbol{U}) \cdot \nabla\} \boldsymbol{u}$. In other words, the Eulerian components of acceleration are frame dependent, but the total Lagrangian acceleration - as a true physical quantity - is, of course, frame independent.

However, our measurements are in a particular Eulerian frame, i.e. attached to the ground. The consequence is that the Lagrangian acceleration, $\boldsymbol{a}$, is a sum of two large quantities, $\boldsymbol{a}_{l}$ and $\boldsymbol{a}_{c}$, due to the contribution of the mean velocity, $\boldsymbol{U}$. Since there is a strong cancellation between $\boldsymbol{a}_{l}$ and $\boldsymbol{a}_{c}$ (and consequently $\boldsymbol{a}$ is much smaller than both $\boldsymbol{a}_{l}$ and $\boldsymbol{a}_{c}$ ) there is a strong 'amplification' of error when evaluating $\boldsymbol{a}$. The same observation is true when looking at $\boldsymbol{a}_{l}^{\prime}$ and $\boldsymbol{a}_{c}^{\prime}$ (which are much smaller than $\boldsymbol{a}_{l}$ and $\boldsymbol{a}_{c}$ owing to removal of the contribution from the mean velocity, $\boldsymbol{U}$ ); both are much more contaminated by noise (in the sense of relative error) than $\boldsymbol{a}_{l}$ and $\boldsymbol{a}_{c}$. The problem of the errors that become especially apparent when we deal with a small quantity, obtained as a difference of large ones, was discussed in Part 1 (Gulitski et al. $2007 a$ ). It is not easy (if even possible) to estimate their impact directly. When results from DNS or other experiments exist, comparison with them can give an indication of that impact.

The main goal is to study the field of Lagrangian accelerations, $\boldsymbol{a} \equiv \mathrm{D} \boldsymbol{u} / \mathrm{D} t$, and its Eulerian components: the local acceleration, $\boldsymbol{a}_{l}=\partial \boldsymbol{u} / \partial t$, and the convective one, $\boldsymbol{a}_{c}=(\boldsymbol{u} \cdot \nabla) \boldsymbol{u}$, as was done for low Reynolds numbers in DNS (Tsinober et al. 2001). This includes the random Taylor (sweeping decorrelation) hypothesis and associated issues of geometrical statistics of accelerations, involving the above mentioned variety of Eulerian components of the total acceleration. In particular, we are interested in mutual (statistical) cancellation between the local acceleration, $\boldsymbol{a}_{l}=\partial \boldsymbol{u} / \partial t$, and the convective one, $\boldsymbol{a}_{c}=(\boldsymbol{u} \cdot \nabla) \boldsymbol{u}$. Since these quantities are vectors, the degree of this mutual cancellation should be studied both in terms of their magnitude and 
the geometry of vector alignments. Among other things, this cancellation should be reflected in that the total acceleration, $\boldsymbol{a}=\boldsymbol{a}_{l}+\boldsymbol{a}_{c}$, is much smaller than its local and convective components, $\boldsymbol{a}_{l}$ and $\boldsymbol{a}_{c}$, in large negative correlation between $\boldsymbol{a}_{l}$ and $\boldsymbol{a}_{c}$, and in strong (anti-)alignment of $\boldsymbol{a}_{l}$ and $\boldsymbol{a}_{c}$.

A natural by-product will be the direct check of the Taylor hypothesis using the new technique mentioned above. We would like to stress that only a technique allowing us to measure simultaneously and independently the spatial and temporal velocity derivatives enables us to address all these issues. It is essential to note that, in principle, neither the random Taylor hypothesis nor the conventional one can be strictly valid.

Other aspects include one- and two-point statistics, conditional statistics of accelerations and its Eulerian components and Reynolds-number effects.

\section{Acceleration variance}

\subsection{General notes}

The acceleration variance, $\left\langle a_{k} a_{k}\right\rangle$, is a key quantity in a number of issues. Following the above cited papers, we use the Kolmogorov normalized version of acceleration variance, $a_{0}=(1 / 3)\left\langle a_{k} a_{k}\right\rangle \epsilon^{-3 / 2} v^{1 / 2}$ (Yaglom 1949; Obukhov \& Yaglom 1951; Monin \& Yaglom 1975). Its value and scaling with the Reynolds number are essential for stochastic Lagrangian models and for Lagrangian probability density function models of turbulent diffusion if these models are to incorporate finite-Reynolds-number effects.

As mentioned, at the present state of the art, the acceleration variance, $\left\langle a_{k} a_{k}\right\rangle$, and consequently $a_{0}$ are not directly obtainable from Eulerian measurements owing to extremely strong cancellation between $\boldsymbol{a}_{l}$ and $\boldsymbol{a}_{c}$. As found by Tsinober et al. (2001) from DNS simulations, the magnitude of the correlation coefficient between $\boldsymbol{a}_{l}$ and $\boldsymbol{a}_{c}$ is increasing with $R e_{\lambda}$ and is already about -0.97 at $R e_{\lambda}=240$. Therefore, we can expect that this correlation coefficient will be extremely close to minus unity at larger $R e_{\lambda}$. Consequently, $\boldsymbol{a}_{l}+\boldsymbol{a}_{c}$ will become much smaller than both $\boldsymbol{a}_{l}$ and $\boldsymbol{a}_{c}$. At $R e_{\lambda}=240$, the RMS of $\boldsymbol{a}$ is already less than 0.1 of both $\boldsymbol{a}_{l}$ and $\boldsymbol{a}_{c}$. Hence $\boldsymbol{a}$, obtained as a sum of $\boldsymbol{a}_{l}$ and $\boldsymbol{a}_{c}$ at large $R e_{\lambda}$, is 'drowning' in noise. It is noteworthy that PTV experiments at low $R e_{\lambda}$ (Lüthi et al. 2005) exhibit the same problem. Namely, the RMS of the fluid particle acceleration obtained as a sum of $\boldsymbol{a}_{l}$ and $\boldsymbol{a}_{c}$ is twice as large as that obtained directly.

Hill \& Wilczak (1995) found a relation determining the acceleration variance via the fourth-order structure functions of the velocity field

$$
\left\langle a_{k} a_{k}\right\rangle=\chi-\frac{35}{2} v\left\langle\left(\partial u_{1} / \partial x_{1}\right)^{3}\right\rangle,
$$

with

$$
\begin{gathered}
\chi=4 \int_{0}^{\infty} r^{-3}\left[S_{u_{1}}^{(4)}(r)+S_{u_{2}}^{(4)}(r)-6 S_{u_{1} u_{2}}^{(4)}(r)\right] \mathrm{d} r \\
S_{u_{1}}^{(4)}(r)=\left\langle\left(\Delta u_{1}\right)^{4}\right\rangle ; S_{u_{2}}^{(4)}(r)=\left\langle\left(\Delta u_{2}\right)^{4}\right\rangle ; S_{u_{1} u_{2}}^{(4)}(r)=\left\langle\left(\Delta u_{1}\right)^{2}\left(\Delta u_{2}\right)^{2}\right\rangle ; \\
\Delta u_{i}=u_{i}(x+r)-u_{i}(x) .
\end{gathered}
$$

This expression was obtained with the only assumption being of local isotropy, so that $\Delta u_{3}$ can be used in (2.2) instead of $\Delta u_{2}$. Hill \& Wilczak (1995) argued also that 


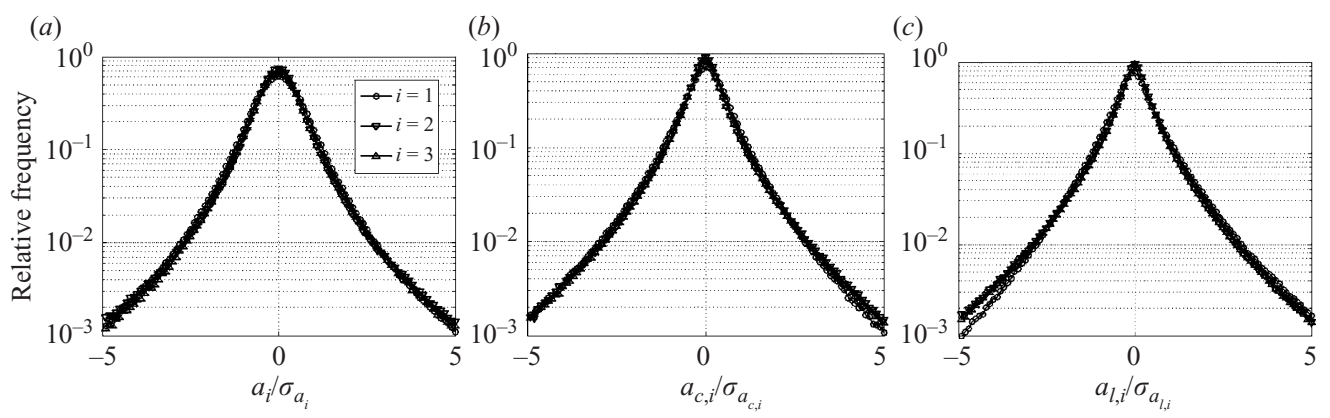

FIGURE 4. PDFs of the $a_{i}, a_{c, i}, a_{l, i}, i=1,2,3$.

a sufficiently precise result can be obtained from

$$
\chi=4 H \chi \int_{0}^{\infty} r^{-3} S_{u_{1}}^{(4)}(r) \mathrm{d} r,
$$

with $H \chi=$ const for sufficiently large Reynolds numbers. Vedula \& Yeung (1999) determined $H \chi$ from DNS and showed that it approaches a constant value of approximately 0.65 for $R e_{\lambda}$ greater than about 200. Also note that assuming isotropy, we obtain

$$
-\frac{35}{2} v\left\langle\left(\partial u_{1} / \partial x_{1}\right)^{3}\right\rangle=v\left\langle\omega_{i} \omega_{j} s_{i j}\right\rangle
$$

This method was implemented in previous attempts to determine acceleration variance using hot-wire anemometry by Hill \& Thoroddsen (1997) and Gylfason et al. (2004).

Figure 2 shows a number of points obtained from our data by the above method. The main feature is that there seems to be no saturation in the $R e$-dependence of $a_{0}$. There are two possibilities. First, this may be a genuine effect. In that case, the scaling proposed by Yaglom (1949) is not 'perfect' and the acceleration variance is larger than that proposed by Yaglom. Secondly, the trend seen in figure 2 is due to the imperfections of the indirect method. The issue seems to be open and requires further more precise measurements.

\section{One-point statistics}

\subsection{PDFs}

Figures 4, 5 and 6 show the PDFs related to accelerations. All the PDFs exhibit strong deviation from Gaussian distributions. This deviation cannot be considered as a pure manifestation of intermittency because acceleration is a nonlinear function of velocity and its derivatives. Therefore, even for a purely Gaussian velocity field we cannot expect the PDFs, associated with accelerations (as any nonlinear functions/functionals of velocity field) to be Gaussian (Tsinober 2001). Indeed, it was shown byTsinober et al. (2001), using the data from DNS, that this is really the case: the PDFs of accelerations of a Gaussian velocity field are strongly non-Gaussian, but somewhat less than those for a real velocity field, resulting from the Navier-Stokes equations.

The PDF of the centred acceleration modulus $a-\langle a\rangle$ (figure $6 a$ ) deserves special mention. It is strongly positively skewed. This behaviour is closely related to the two-point statistics of acceleration moduli. 
(a)

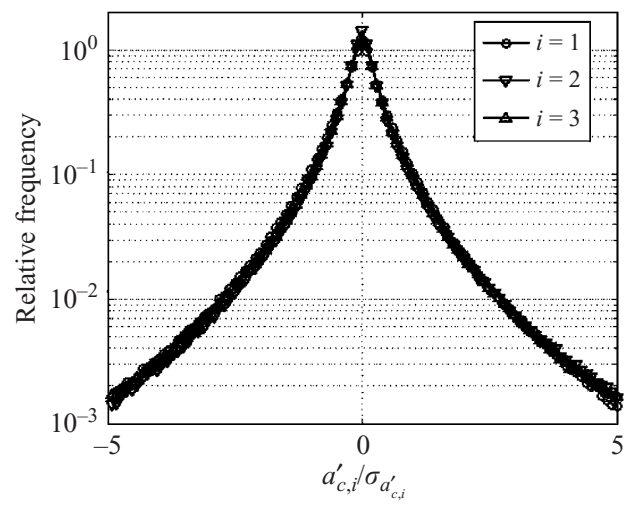

(b)

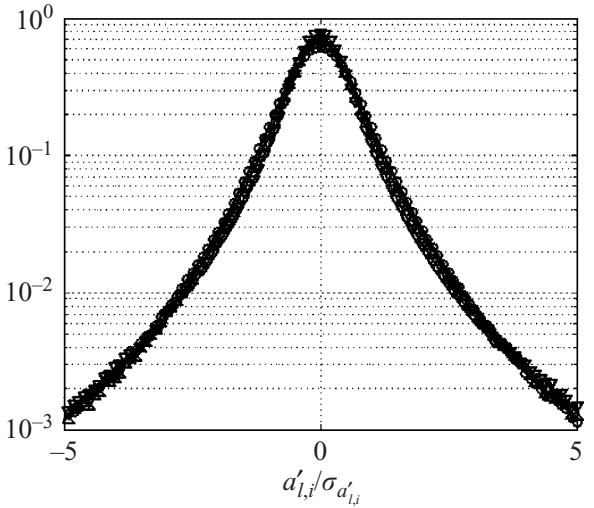

FIGURE 5. PDFs of the $a_{c, i}^{\prime}, a_{l, i}^{\prime}, i=1,2,3$.
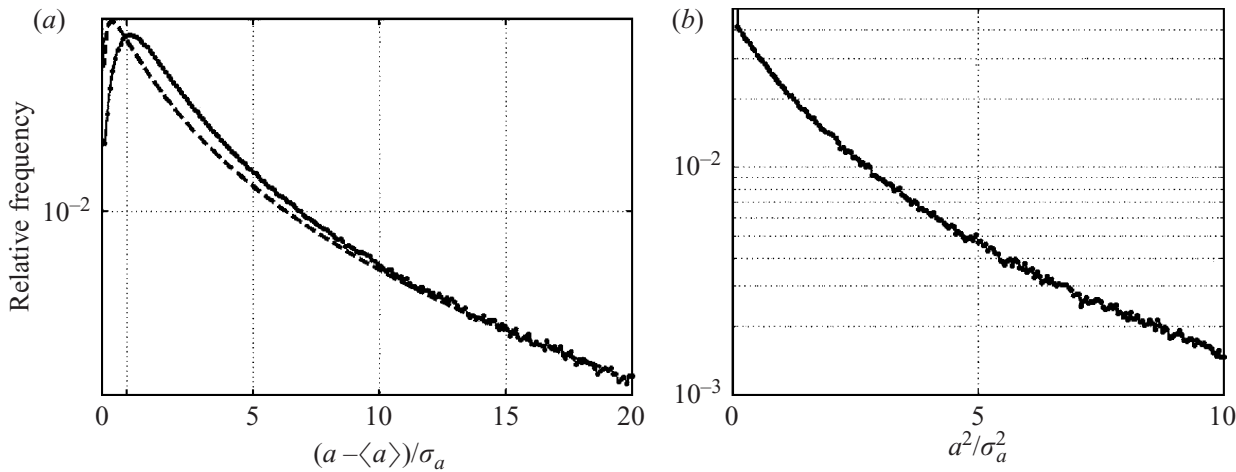

FIgURE 6. (a) PDF of $a-\langle a\rangle$ (solid line). For comparison we show also the PDF for log-normal distribution with variance 1 , shifted to the left by $\langle a\rangle / \sigma_{a}$ (dotted line). (b) PDF of $a^{2}=a_{k} a_{k}$.

\subsection{Geometrical statistics}

By the term geometrical statistics, we denote alignments between a variety of vectors.

\subsubsection{Random Taylor hypothesis}

These issues take their origin from Taylor (1935) and are known as the Taylor hypothesis. Here we demonstrate one of the basic alignments associated with the random Taylor hypothesis (or the sweeping decorrelation hypothesis) which concerns the relations between the instantaneous Lagrangian acceleration and its (Eulerian) 'components' $\boldsymbol{a}_{l}=\partial \boldsymbol{u} / \partial t$ and $\boldsymbol{a}_{c}=(\boldsymbol{u} \cdot \nabla) \boldsymbol{u}$. It was suggested by Tennekes (1975) that

in turbulence with high Reynolds numbers... the dissipative eddies flow past an Eulerian observer in a time much shorter than the time scale which characterizes their own dynamics. This suggests that Taylor's 'frozen-turbulence' approximation should be valid for the analysis of the consequences of large-scale advection of the turbulent microstructure.

The outcome is that (i) there should be a strong cancellation effect between $\boldsymbol{a}_{l}$ and $\boldsymbol{a}_{c}$, so that $\boldsymbol{a}=\boldsymbol{a}_{l}+\boldsymbol{a}_{c}$ is much smaller than both $\boldsymbol{a}_{l}$ and $\boldsymbol{a}_{c}$ (e.g. comparing their variances), and (ii) $\boldsymbol{a}_{l}$ and $\boldsymbol{a}_{c}$ should be strongly anti-aligned. Both effects were convincingly demonstrated by Tsinober et al. (2001) with DNS data for relatively 


$\begin{array}{cccccc}\text { Variance } & \boldsymbol{a} & \boldsymbol{a}_{l} & \boldsymbol{a}_{c} & \boldsymbol{a}_{l}^{\prime} & \boldsymbol{a}_{c}^{\prime} \\ 9900 & 39900 & 60100 & 9100 & 2600\end{array}$

TABLE 1. Variances in $\mathrm{m} \mathrm{s}^{-2}$ of $\boldsymbol{a}, \boldsymbol{a}_{l}, \boldsymbol{a}_{c}, \boldsymbol{a}_{l}^{\prime}, \boldsymbol{a}_{c}^{\prime}$.

(a)

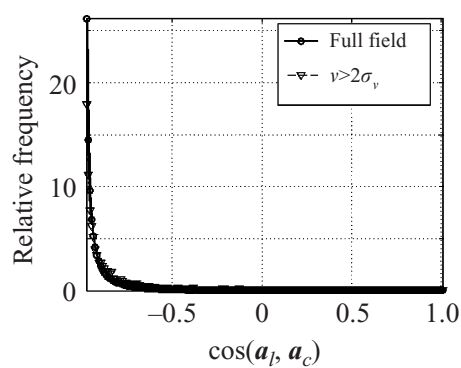

(b)

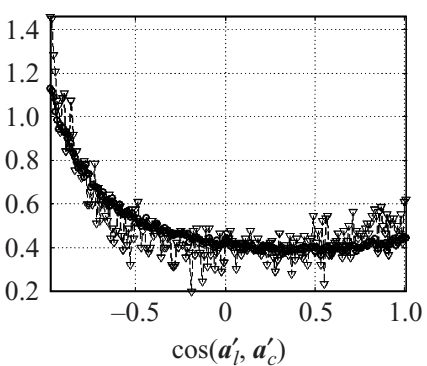

(c)

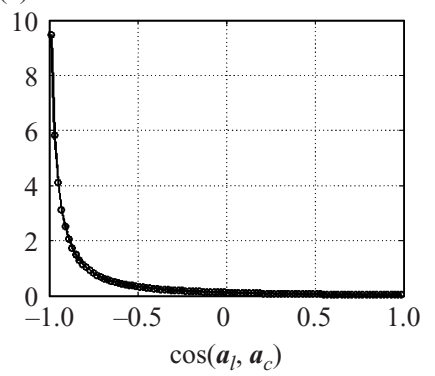

FiguRE 7. PDFs of $\cos \left(\boldsymbol{a}_{l}, \boldsymbol{a}_{c}\right)$ in $(a)$ the frame attached to the ground and $(b)$ in the frame moving with the mean velocity from the field experiment; $(c)$ PDF of $\cos \left(\boldsymbol{a}_{l}, \boldsymbol{a}_{c}\right)$ from PTV experiment.
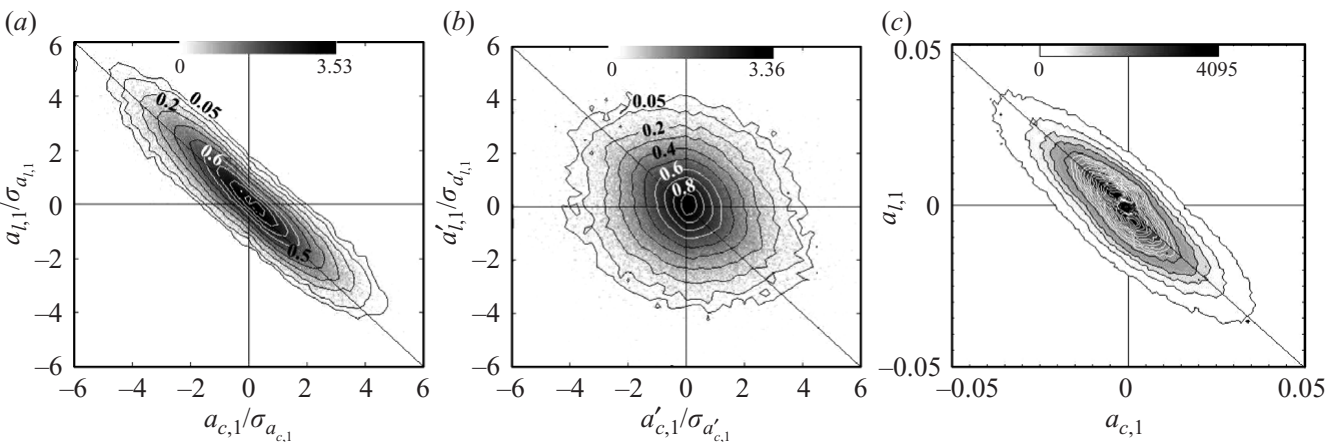

FIGURE 8. Joint PDFs of $x_{1}$-components of $\boldsymbol{a}_{l}$ and $\boldsymbol{a}_{c}(a)$ in the frame attached to the ground and $(b)$ in the frame moving with the mean velocity from field experiment; $(c)$ joint PDF from PTV experiment. (a) Correlation coefficient $=-0.926,(b)-0.193$.

small $R e_{\lambda} \leqq 400$ and with preliminary experiments (Kholmyansky et al. 2001b). We observed these effects at $R e_{\lambda} \sim 10^{4}$.

The results are shown in table 1 with variances of $\boldsymbol{a}, \boldsymbol{a}_{l}, \boldsymbol{a}_{c}, \boldsymbol{a}_{l}^{\prime}, \boldsymbol{a}_{c}^{\prime}$ and in figure 7 with an example of the PDFs of $\cos \left(\boldsymbol{a}_{l}, \boldsymbol{a}_{c}\right), \cos \left(\boldsymbol{a}_{l}^{\prime}, \boldsymbol{a}_{c}^{\prime}\right)$. Both clearly exhibit the two aspects mentioned above. The anti-alignment is observed also for the acceleration components associated with the frame moving with the mean velocity, i.e. $\cos \left(\boldsymbol{a}_{l}^{\prime}, \boldsymbol{a}_{c}^{\prime}\right)$, though in a much weaker form, most probably due to the large relative error in these quantities.

Another view on the above is shown in figure 8 with the joint PDFs of Cartesian components of local and convective accelerations (only those for the $x_{1}$-component as the $x_{2}$ - and $x_{3}$-components exhibit similar behaviour) for both acceleration 
components in the frame attached to the ground and the frame moving with the mean velocity. The correlation between acceleration components in the frame, attached to the ground, is pretty high, whereas the correlation in the frame, moving with the mean velocity, is rather weak because of a large relative error for these quantities.

Our main focus here is on the original random Taylor hypothesis concerning the local and convective accelerations. However, it appears that the random Taylor hypothesis is valid not only for the velocity field, but for a variety of other quantities such as velocity derivatives (vorticity and strain), temperature and its gradient, and solenoidal passive vectors. In other words, the random Taylor hypothesis has a universal nature, which is manifested in a strong tendency for cancellation between the local temporal derivative, $\partial Q / \partial t$, and the advective derivative, $u_{k} \partial Q / \partial x_{k}$, of whatever quantity, $Q$. Thus the full material (Lagrangian) derivative, $\mathrm{D} Q / \mathrm{D} t=\partial Q / \partial t+u_{k} \partial Q / \partial x_{k}$, is much smaller (at least an order of magnitude) than its Eulerian components. Evidence regarding this issue is given by Galanti et al. (2003).

\subsubsection{Alignments with the eigenframe, $\lambda_{k}$, of the rate of strain tensor, $s_{i j}$.}

The alignments are shown in figures 9-12. The first important feature of all the alignments with the eigenframe, $\lambda_{k}$, of the rate of strain tensor, $s_{i j}$, is that they are all similar for the quantities, associated with the frame attached to the ground, and for those in the frame moving with the mean velocity; in several cases they are very similar. This is a clear indication that the quantities, associated with the frame moving with the mean velocity, are captured correctly in spite of a much larger error in their estimates as explained above.

As well as the important technical aspects described, we provide comments on the nature of the alignments shown as follows.

One of the common features is that all the vectors tend to be normal to the intermediate eigenvector, $\boldsymbol{\lambda}_{2}$, of the rate of strain tensor and tend to be aligned with both the pure stretching eigenvector, $\boldsymbol{\lambda}_{1}$, and the pure compression eigenvector, $\lambda_{3}$. This is consistent with intuition, based on the fact that vorticity is strongly aligned with $\lambda_{2}$ and the expectation that fluid particle acceleration is mostly normal to vorticity and consequently aligned with the pure stretching and compressing eigen-directions of the rate-of-strain tensor (see figure 13).

The alignments, obtained from hot-wire measurements, are similar to those observed in low-Reynolds-number experiments using three-dimensional particle tracking velocimetry by Lüthi et al. (2005). Examples of such alignments are shown in figure 9.

\subsubsection{Additional significant alignments}

As mentioned, there are various physically meaningful Eulerian components of acceleration: $\boldsymbol{a}=\boldsymbol{a}_{l}+\boldsymbol{a}_{c}=\boldsymbol{a}_{l}+\boldsymbol{a}_{L}+\boldsymbol{a}_{B}=\boldsymbol{a}_{1}+\boldsymbol{a}_{\perp}$, where $\boldsymbol{a}_{c}=(\boldsymbol{u} \cdot \nabla) \boldsymbol{u}$ is the convective acceleration and $\boldsymbol{a}_{l}=\partial \boldsymbol{u} / \partial t$ is the local acceleration in the frame attached to the ground, $\boldsymbol{a}_{L}=\boldsymbol{\omega} \times \boldsymbol{u}$ is the Lamb vector, $\boldsymbol{a}_{B}=\nabla \boldsymbol{u}^{2} / 2$ is the Bernoulli component of acceleration, $\boldsymbol{a}_{\|}=(\boldsymbol{a} \cdot \widehat{\boldsymbol{u}}) \widehat{\boldsymbol{u}}$ is the acceleration component parallel to the velocity vector, where $\widehat{\boldsymbol{u}}=\boldsymbol{u} / u$ is the unit vector along the velocity vector, and $\boldsymbol{a}_{\perp}=\boldsymbol{a}-\boldsymbol{a}_{\|}$is the acceleration component normal to the velocity vector; similar quantities are defined in the frame moving with the mean velocity. The availability of these quantities allows us to address a rich variety of alignments. 
(a)

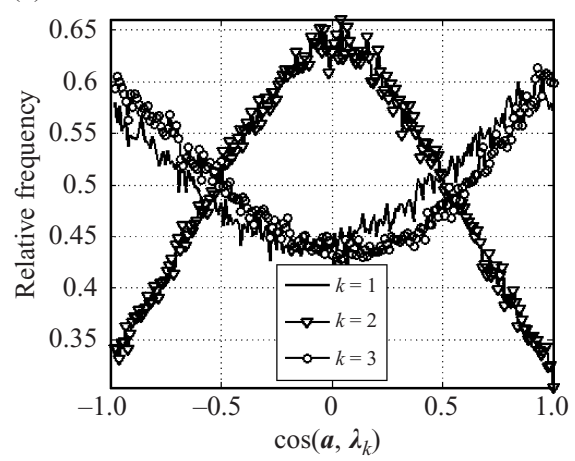

(c)

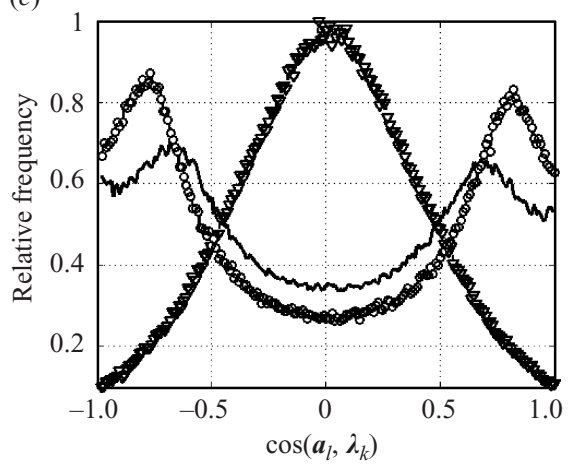

(e)

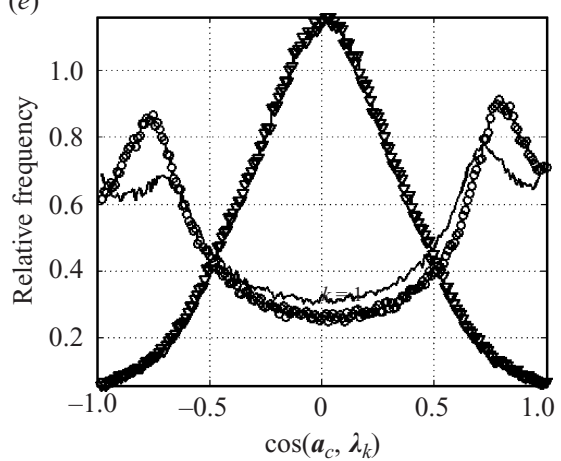

(b)

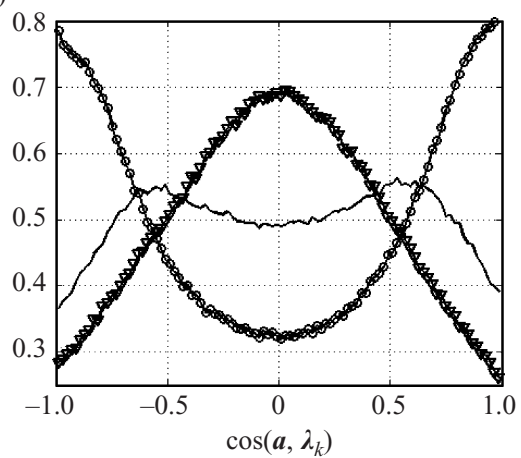

(d)

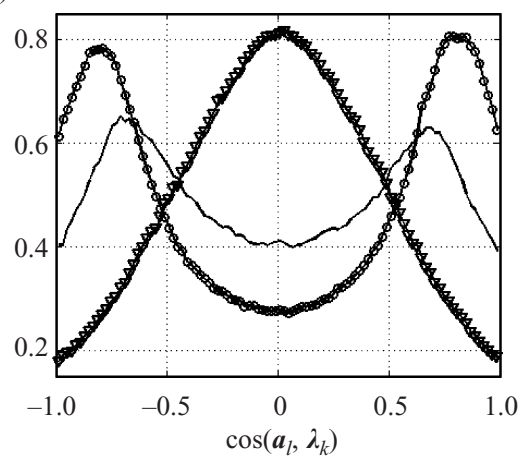

(f)

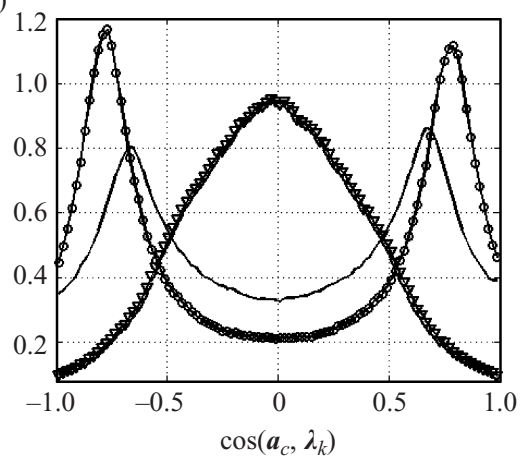

FIGURE 9. PDFs of the cosine of the angle between the acceleration components, $\boldsymbol{a}, \boldsymbol{a}_{l}, \boldsymbol{a}_{c}$, and the eigenframe, $\lambda_{k}$, of the rate of strain tensor, $s_{i j}$, obtained at $R e_{\lambda} \sim 10^{4}$ in our field experiment $(a, c, e)$ and at $R e_{\lambda} \sim 10^{2}$ in the PTV experiment $(b, d, f)$ by Lüthi et al. (2005).

First, here again, we point to the similarity between the alignments involving quantities associated with the frame attached to the ground and quantities in the frame moving with the mean velocity. We show those pairs of figures in which strong alignments are observed.

Secondly, it is of special interest that the alignments shown above are similar also to those observed in low-Reynolds-number experiments using three-dimensional particle tracking velocimetry in experiments by Lüthi et al. (2005). Examples of such alignments are shown in figures 14-20. 
(a)

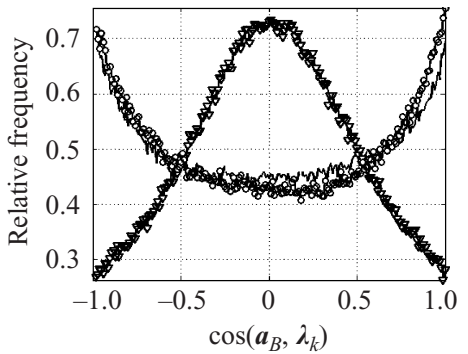

(b)

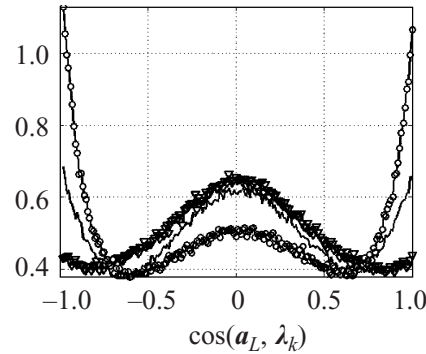

(c)

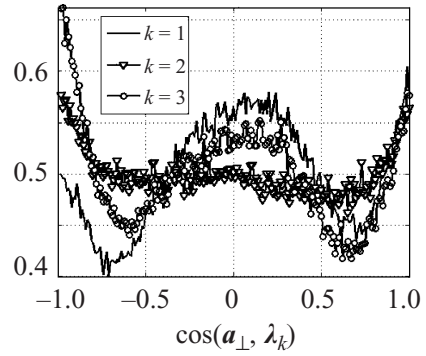

Figure 10. Alignments of the acceleration components, $\boldsymbol{a}_{B}, \boldsymbol{a}_{L}, \boldsymbol{a}_{\perp}$, with the eigenframe, $\boldsymbol{\lambda}_{k}$, of the rate-of-strain tensor, $s_{i j}$, associated with the frame attached to the ground.

(a)

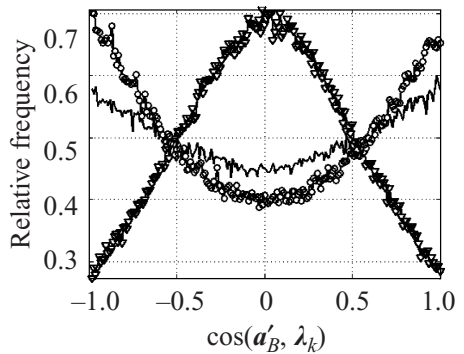

(b)

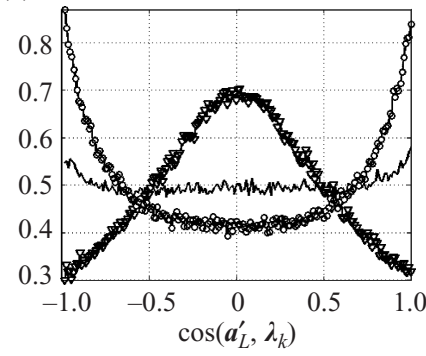

(c)

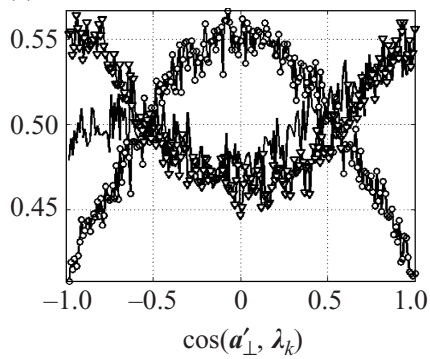

FIGURE 11. Same as in figure 10, associated with the frame moving with the mean velocity.

(a)

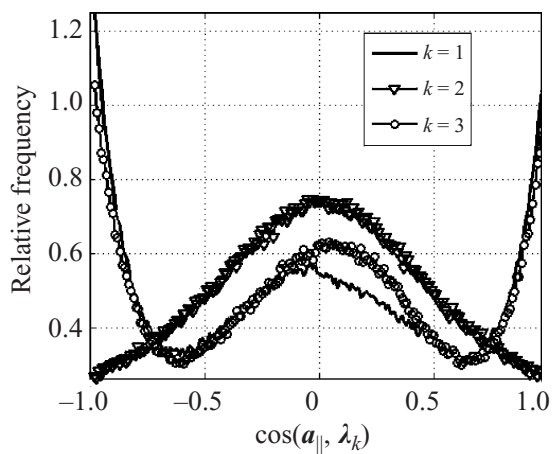

(b)

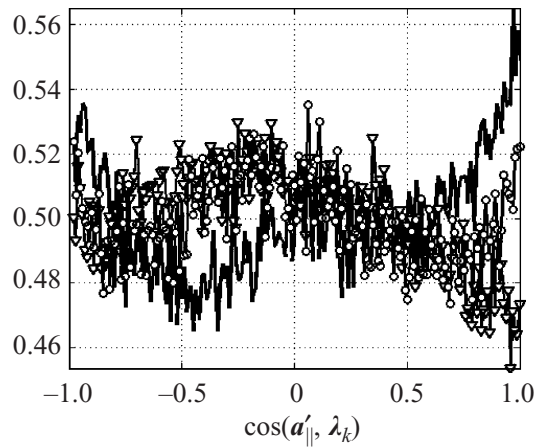

FIGURE 12. Alignments of the acceleration component, $\boldsymbol{a}_{\|}$, with the eigenframe, $\lambda_{k}$, of the rate-of-strain tensor, $s_{i j}$, associated with $(a)$ the frame attached to the ground and $(b)$ the frame moving with the mean velocity.

\section{Two-point statistics}

The auto-correlations of vectors $\boldsymbol{a}, \boldsymbol{a}_{c}, \boldsymbol{a}_{l}, \boldsymbol{a}_{c}^{\prime}$ and $\boldsymbol{a}_{l}^{\prime}\left(\operatorname{such}\right.$ as $\left.\left\langle\boldsymbol{a}_{x_{i}}(x+r) \cdot \boldsymbol{a}_{x_{i}}(x)\right\rangle\right)$ and their Cartesian components (such as $\left.\left\langle\boldsymbol{a}_{x_{1}}(x+r) \cdot \boldsymbol{a}_{x_{1}}(x)\right\rangle\right)$ and the modulus of $\boldsymbol{a}$ are shown in figures 21 and 22 along with mean values of the cosine of the angle between corresponding vectors at two positions $\langle\cos \{\boldsymbol{a}(x+r), \boldsymbol{a}(x)\}\rangle$, (figure 23). Here the values of the distance, $r$, between the two points with sequential numbers $i$ and $i+n$ is calculated as $r=n \Delta t\left\langle u_{1}\right\rangle$, where $\Delta t=10^{-4} \mathrm{~s}$ is the sampling time. 
(a)

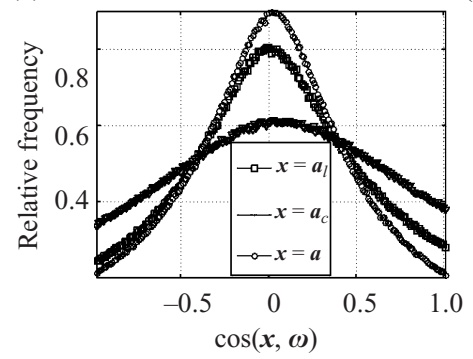

(b)

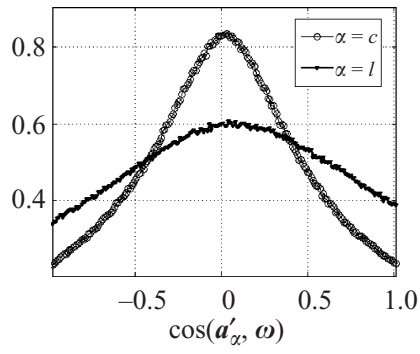

(c)

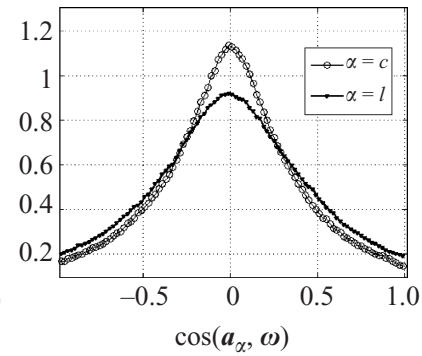

FIGURE 13. PDFs of the cosine of the angle between acceleration, $\boldsymbol{a}$, and vorticity, $\boldsymbol{\omega},(a)$ in the frame attached to the ground and $(b)$ in the frame moving with the mean velocity from field experiment; $(c)$ similar PDF from PTV experiment.

(a)

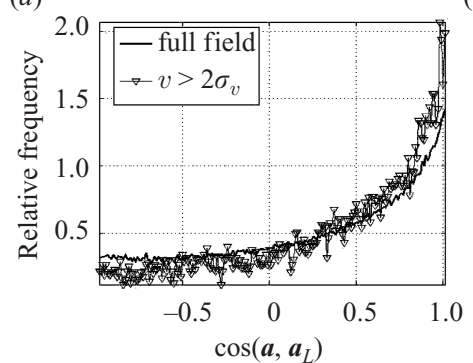

(b)

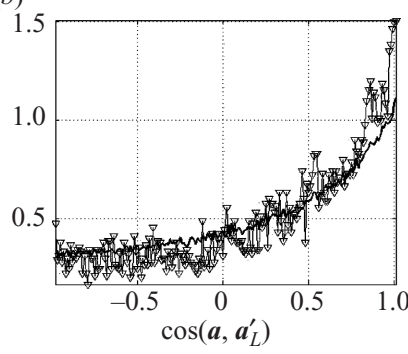

(c)

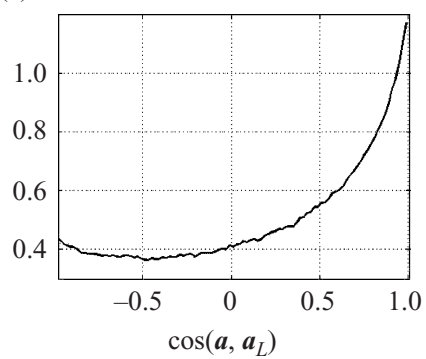

Figure 14. Same as in figure 13 for $\cos \left(\boldsymbol{a}, \boldsymbol{a}_{L}\right)$.

(a)

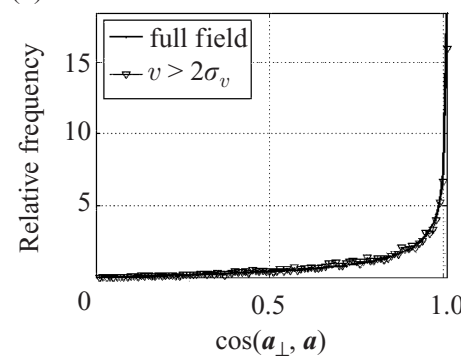

(b)

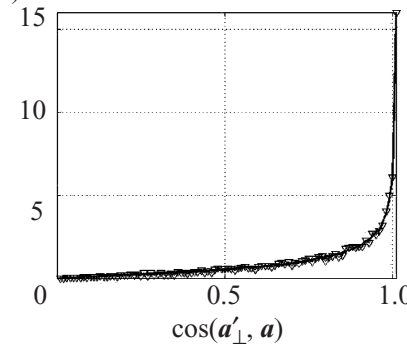

(c)

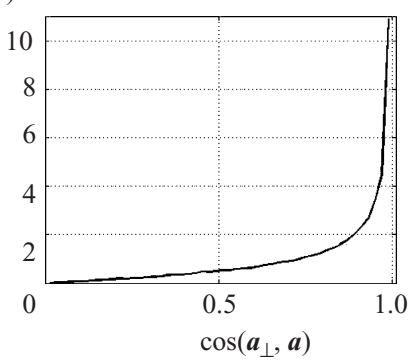

FIGURE 15. Same as in figure 13 for $\cos \left(\boldsymbol{a}, \boldsymbol{a}_{\perp}\right)$.

(a)

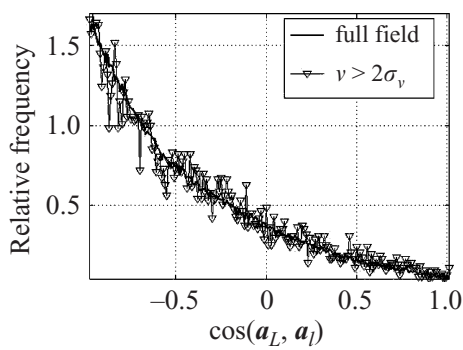

(b)

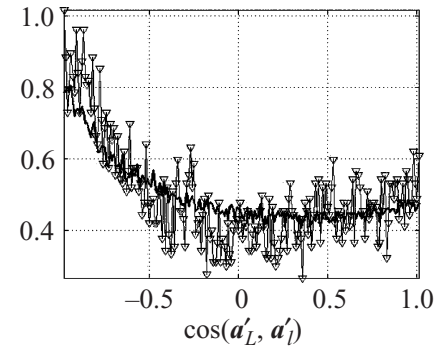

(c)

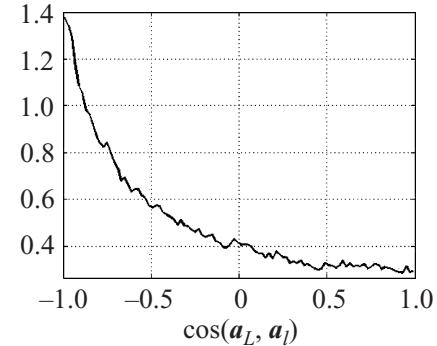

FIgURE 16. PDFs of $\cos \left(\boldsymbol{a}_{L}, \boldsymbol{a}_{l}\right)(a)$ in the frame attached to the ground and $(b)$ in the frame moving with the mean velocity, field experiment; $(c)$ similar PDF from PTV experiment. 
(a)

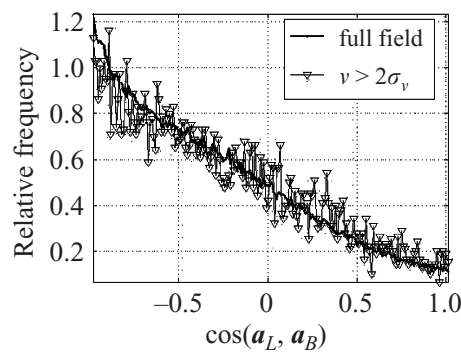

(b)

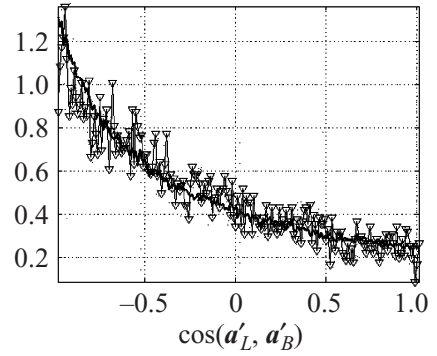

(c)

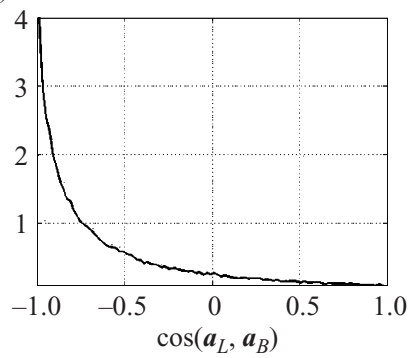

Figure 17. Same as in figure 16 for $\cos \left(\boldsymbol{a}_{L}, \boldsymbol{a}_{B}\right)$.

(a)

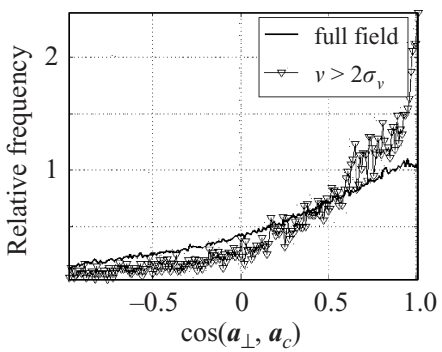

(b)

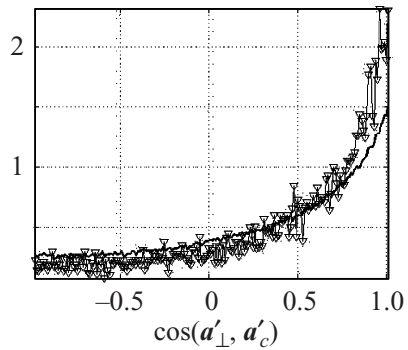

(c)

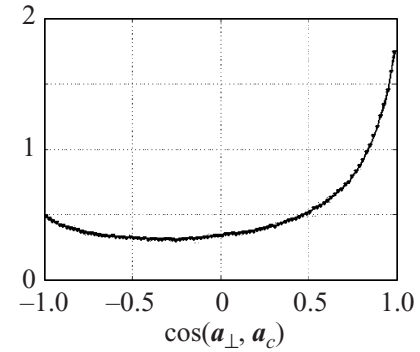

FIGURE 18. Same as in figure 16 for $\cos \left(\boldsymbol{a}_{\perp}, \boldsymbol{a}_{c}\right)$.

(a)

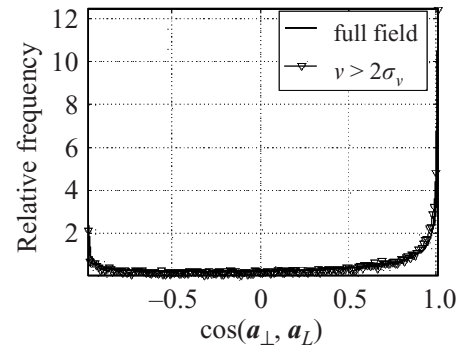

(b)

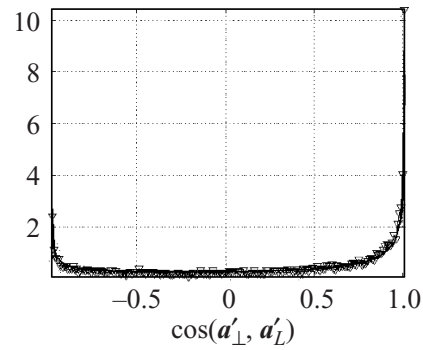

(c)

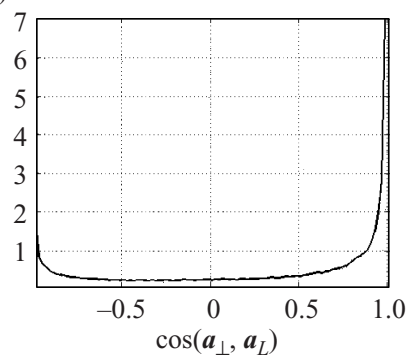

FIgURE 19. PDFs of $\cos \left(\boldsymbol{a}_{\perp}, \boldsymbol{a}_{L}\right)(a)$ in the frame attached to the ground and $(b)$ in the frame moving with the mean velocity, field experiment; $(c)$ similar PDF from PTV experiment.

(a)

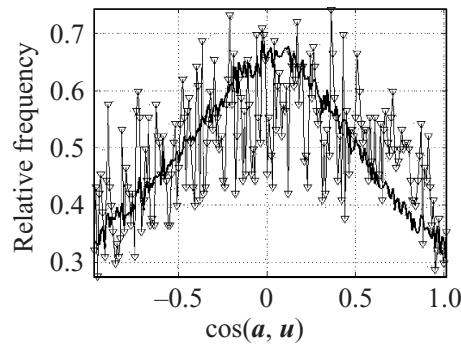

(b)

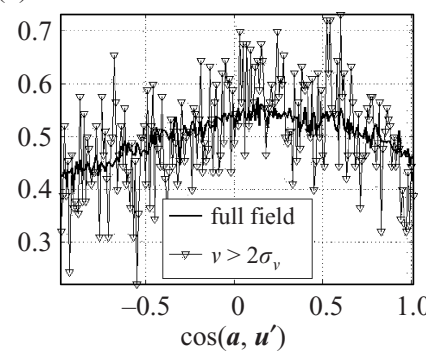

(c)

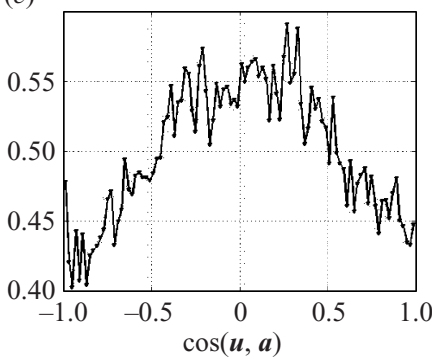

FIGURE 20. PDFs of the cosine of the angle between the acceleration, $\boldsymbol{a}$, and velocity, $\boldsymbol{u},(a)$ in the frame attached to the ground and $(b)$ in the frame moving with the mean velocity from field experiment; $(c)$ similar PDF from PTV experiment. 

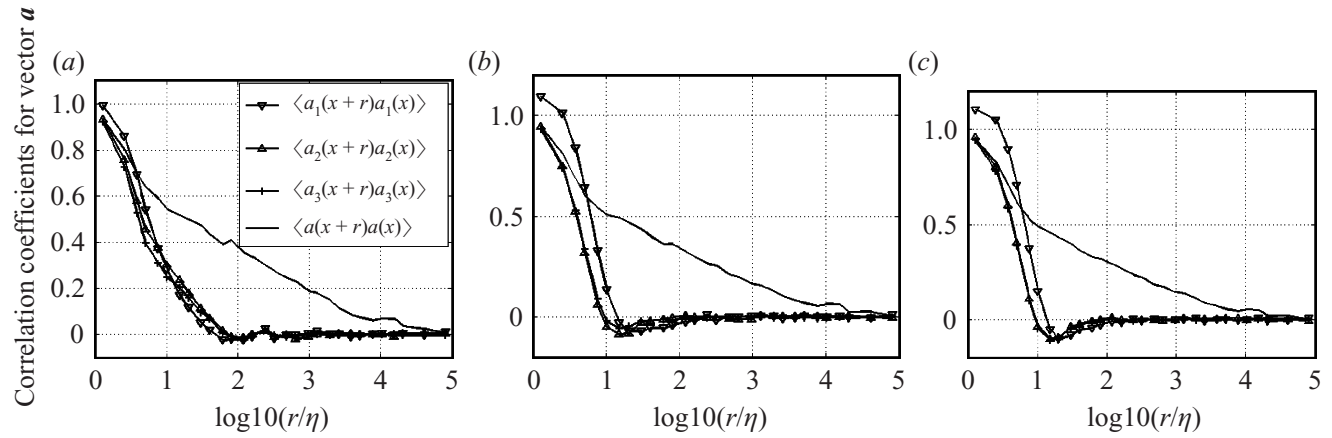

FIGURE 21. The auto-correlations of vectors $(a) \boldsymbol{a},(b) \boldsymbol{a}_{c},(c) \boldsymbol{a}_{l}$ and their Cartesian components from field experiment.
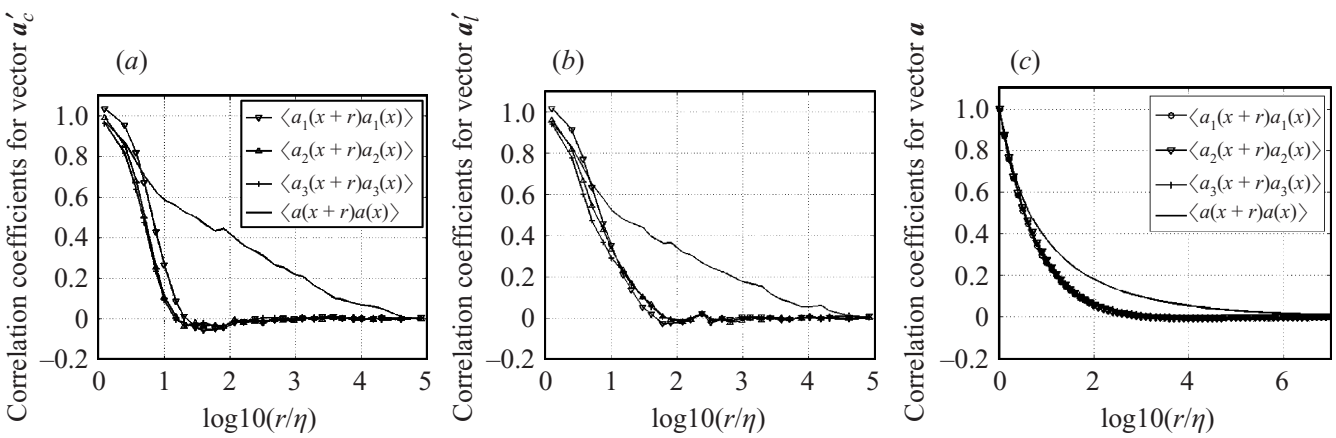

FIgURE 22. The auto-correlations of vectors $(a) \boldsymbol{a}_{c}^{\prime},(b) \boldsymbol{a}_{l}^{\prime}$ from field experiment. (c) The auto-correlations of vectors $\boldsymbol{a}$ from PTV experiment.
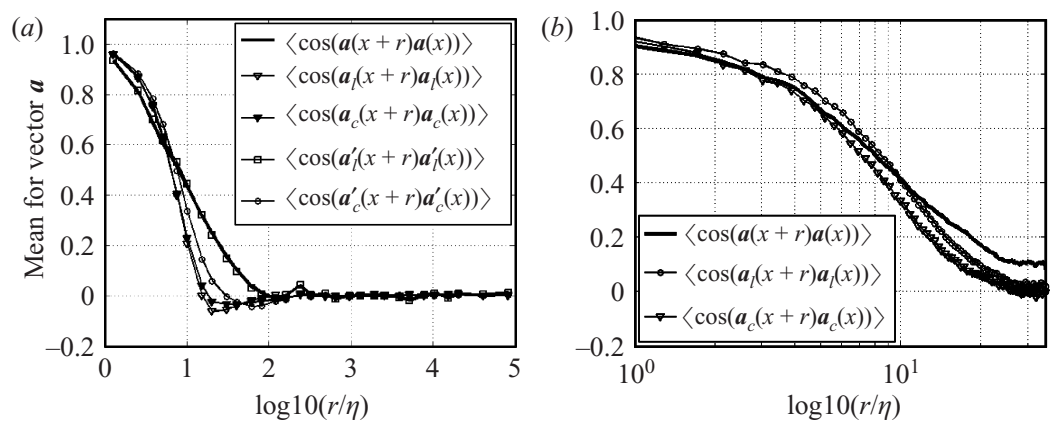

FiguRE 23. Mean values of the cosine of the angle between corresponding vectors at two positions, $\langle\cos \{\boldsymbol{a}(x+r), \boldsymbol{a}(x)\}\rangle(a)$ from field experiment, and $(b)$ from PTV experiment.

The main features are similar to those obtained for Lagrangian correlations both in DNS (Yeung 1997) and experimentally (Mordant et al. 2004a). The first is that all the vectors and their components are correlated over much shorter distances - typically 10 Kolmogorov lengths - than their moduli. The latter are correlated over large distances of the order of $10^{4}$ Kolmogorov lengths, which is comparable with the integral scale as for Lagrangian correlations both in DNS (Yeung 1997) and experiments (Mordant et al. 2004a). This behaviour is observed also in PTV experiments by Lüthi et al. (2005) (figure 22c). 

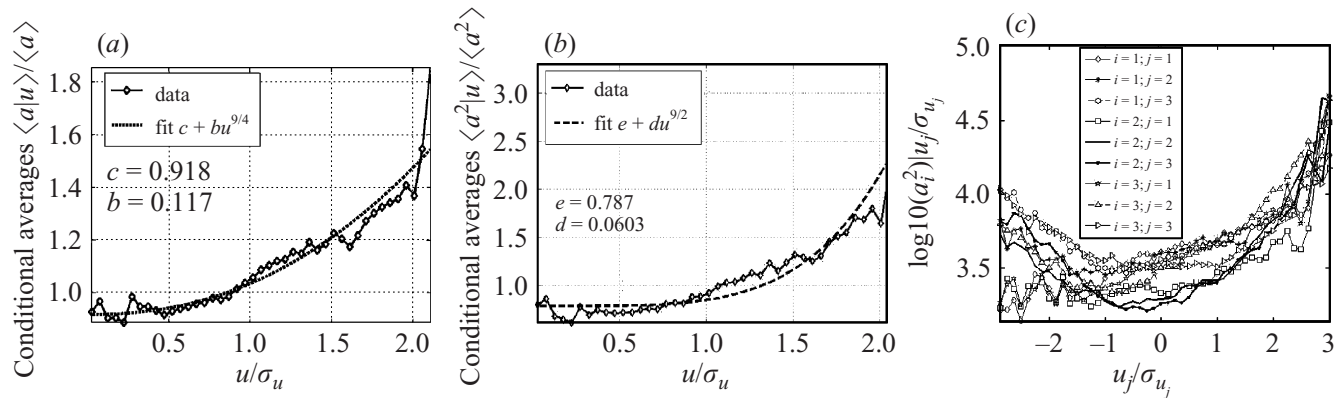

FIGURE 24. Conditional averages of $(a) a$ and $(b) a^{2}$ on the magnitude of the vector of velocity fluctuations, $u$, and of $a_{i}^{2}$ on $u_{j}$, where $i, j=1,2,3(c)$. The fits are as implied by simple arguments of Aringazin \& Mazhitov (2004) and used later by Crawford et al. (2005). Note that in Crawford et al. (2005) $e \sim 2$ and $d \sim 10^{-2}$ and is Reynolds dependent.

A similar feature is found for the cosines of the corresponding angles (figure 23), though the difference is much smaller. The above feature is the same both for acceleration components in the frame attached to the ground and in the frame moving with the mean velocity (figure $22 a, b$ ).

The behaviour of second-order structure functions of Cartesian components of $\boldsymbol{a}$, $\boldsymbol{a}_{c}, \boldsymbol{a}_{l}, \boldsymbol{a}_{c}^{\prime}$ and $\boldsymbol{a}_{l}^{\prime}$ is consistent with the results on correlations shown above.

\section{Conditional statistics, non-locality}

Fluid particle accelerations are considered as small-scale quantities. The long-range correlations of the moduli of accelerations reported above and those by previous authors can be considered as a kind of manifestation of non-locality. This issue is addressed in the present section.

We have seen in Part 1 (Gulitski et al. 2007a), that the statistics of derivatives (i.e. small-scale quantities) is not independent of large-scale quantities, as is usually assumed in a variety of theoretical approaches. Similar results were obtained by Sawford et al. (2003); Mordant et al. (2004a); Crawford et al. (2005) for Lagrangian statistics of accelerations. Our results exhibit the same feature. Namely, the conditional statistics of accelerations are strongly dependent on velocity (figure 24). This can be considered as an additional clear indication of direct coupling between large and small scales and non-locality of turbulent flows. This means that it would be too optimistic to expect quantitative universality of the acceleration statistics, e.g. such as those shown in figure $24(a, b)$ among others. Hence the fits shown in these figures (implied by simple arguments of Aringazin \& Mazhitov (2004) and used later by Crawford et al. (2005)) cannot be considered much more than just fits. Nevertheless, they reflect the qualitative trends correctly and are the same in Crawford et al. (2005) and in figure $24(a, b)$. However, they are very different quantitatively, as should be expected, since the large-scale properties (and Reynolds numbers) of the flow studied by Crawford et al. (2005) (French washing machine) are qualitatively and essentially different from those in the atmospheric surface layer addressed in this study. Therefore, it does not make much sense (if at all) to look for quantitative comparison between the two.

Our observations are made in Euler frames; nevertheless, we observed the same tendency of acceleration moduli to be correlated over large distances of the order of $10^{4}$ Kolmogorov lengths, which is comparable with the integral scale, just as for Lagrangian correlations. 


\section{Conclusions and possible future directions}

The main technical achievement is the possibility of employing the multi-hot-wire technique without invoking the Taylor hypothesis and thereby accessing the fluid particle acceleration and a variety of its Eulerian components. This required the design and manufacturing of a special probe and introduction of modifications in the existing system.

The new technique allowed us to obtain a number of previously inaccessible results. Our results prove the feasibility of correct measurements of the streamwise derivatives without invoking the Taylor hypothesis, thus allowing us to address many important issues associated with accelerations and related matters. These concern a number of properties of fluid particle accelerations and its Eulerian components such as variances, geometrical statistics of accelerations, random Taylor (sweeping decorrelation) hypothesis, conditional statistics and non-locality. In particular, it was shown that the random Taylor hypothesis is valid both in the system of coordinates attached to the ground and in the system moving with the mean velocity. In the latter case, the results can be regarded as qualitative only, owing to large relative error. The strong cancellation effects between the local and convective accelerations do not allow us to obtain reliably, for example, the variance of the Lagrangian acceleration. It will be a challenge to improve the quality of measurements and requires non-trivial investment.

The results conform with and confirm one of the main conclusions of Kholmyansky \& Tsinober (2000) and Kholmyansky et al. (2001a,b) that the basic physics of turbulent flow at high Reynolds number $R e_{\lambda} \sim 10^{4}$, at least qualitatively, is the same as at moderate Reynolds numbers, $R e_{\lambda} \sim 10^{2}$. This appears to be true not only for such basic processes as enstrophy and strain production, geometrical statistics, the role of concentrated vorticity and strain, reduction of nonlinearity and non-locality, but also with respect to a variety of issues concerning accelerations and their Eulerian components.

It is important to emphasize, that our claim that "the basic physics of turbulent flow at high Reynolds number $R e_{\lambda} \sim 10^{4}$, at least qualitatively, is the same as at moderate Reynolds numbers, $R e_{\lambda} \sim 10^{2}$ " does not mean that what is called 'Reynolds-number dependence' is unimportant. An immediate example comes from the indirect evaluation of the acceleration variance. It gives clear indications that - if scaled as proposed by Yaglom (1949) - it exhibits a definite Re-dependence and does not saturate at least up to $R e_{\lambda} \sim 10^{4}$. Another well-known example is the behaviour of flatness of individual velocity derivatives and similar quantities based on vorticity and/or strain.

Reynolds dependence is of extreme importance in a great variety of purely engineering problems and other applications. It is important in basic issues of asymptotic behaviour and limiting state(s) of turbulent flows as $R e \rightarrow \infty$. It remains to classify and distinguish between Reynolds-dependent and Reynolds-independent quantities/phenomena in turbulence. At this stage we hold the opinion, that at a qualitative level the basic physics of turbulence is Reynolds-number independent.

One of the main technical challenges for the future is the reduction of the relative error for the acceleration components in the system moving with the mean velocity. This requires substantial improvement of the system such as (i) further miniaturization of the probe design both with respect to its individual arrays and to the whole probe in order to minimize the influence of velocity gradients across the individual arrays and the whole probe, and (ii) further improvement of the calibration system. It is possible to build a probe of the overall scale only slightly larger than $1 \mathrm{~mm}$. It can be done with the available technology on the basis of miniature arrays (figure 25). 


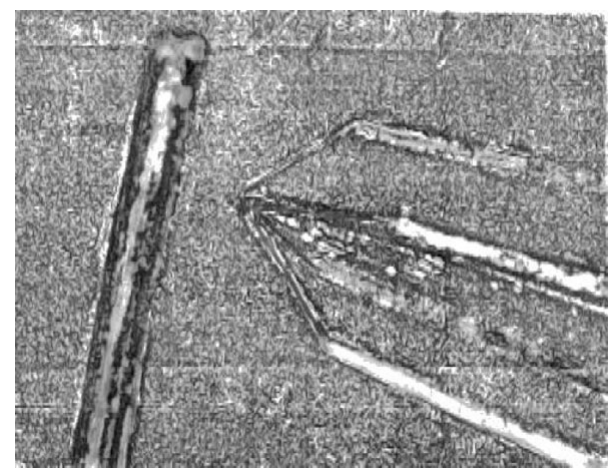

Figure 25. An array $(0.35 \mathrm{~mm}$ at the tip) built with a Pt-10\% Rho wire, $1.25 \mu \mathrm{m}$ in diameter. A human hair $(<70 \mu \mathrm{m}$ in diameter $)$ is shown on the left-hand side.

This work was supported in part by the Israel Science Foundation (ISF), founded by the Israel Academy of Sciences and Humanities, Research grant 34/02; the United States - Israel Binational Science Foundation (BSF), No. 2002264. The field experiment in Switzerland was supported by the Vice President for Research of ETH, Zürich.

The authors appreciate the assistance provided by $\mathrm{Dr} \mathrm{K}$. W. Hoyer and $\mathrm{Mr} \mathrm{T}$. Blunschi from ETH.

The authors gratefully acknowledge the particle tracking data provided by the group of J. Mann, S. Ott and J. Berg from Risø National Laboratory, Denmark.

\section{REFERENCES}

Aringazin, A. K. \& Mazhitov, M. I. 2004 Stochastic models of Lagrangian acceleration of fluid particle in developed turbulence. Intl J. Mod. Phys. B18, 3095-3168.

Bernard, P. S., Thomas, J. M. \& Handler, R. A. 1993 Vortex dynamics and the production of Reynolds stress. J. Fluid Mech. 253, 385-419.

Biferale, L., Boffetta, G., Celani, A., Devenish, B. J., Lanotte, A. \& Toschi, F. 2004 Multifractal statistics of Lagrangian velocity and acceleration in turbulence. Phys. Rev. Lett. 93, 064502/1-4.

Borgas, M. S. \& SAWford, B. L. 1991 The small-scale structure of acceleration correlations and its role in the statistical theory of turbulent dispersion. J. Fluid Mech. 228, 295-320.

Busen, R., Gulitsky, G., Kholmyansky, M., Schumann, U., Tsinober, A. \& Yorish, S. 2001 An airborne experiment on turbulent velocity derivatives. Final Report for the German Israeli Foundation, Grant no. I-541-132.08/97.

Chen, L., Goto, S. \& Vassilicos, J. C. 2006 Turbulent clustering of stagnation points and inertial particles. J. Fluid Mech. 553, 143-154.

Christensen, K. T. \& Adrian, R. J. 2001 The small-scale structure of acceleration in turbulent channel flow. In Proceedings of the Second International Symposium on Turbulence and Shear Flow Phenomena, Stockholm, June, 27-29, 2001 (ed. E. Lindborg, A. Johansson, J. Eaton, J. Humphrey, N. Kasagi, M. Leschziner \& M. Sommerfeld), vol. 1, pp. 21-26.

Christensen, K. T. \& Adrian, R. J. $2002 a$ Measurements of instantaneous Eulerian acceleration fields by particle image accelerometry: method and accuracy. Exps. Fluids 33, 759-769.

Christensen, K. T. \& Adrian, R. J. 2002b The velocity and acceleration signatures of small-scale vortices in turbulent channel flow. $J$. Turbulence 3, 023/1-28.

Crawford, A. M., Mordant, N. \& Bodenschatz, E. 2005 Joint statistics of the Lagrangian acceleration and velocity in fully developed turbulence. Phys. Rev. Lett. 94, 024501/1-4.

Dahm, W. J. A. \& Southerland, K. B. 1997 Experimental assessment of Taylor's hypothesis and its applicability to dissipation estimates in turbulent flows. Phys. Fluids 9, 2101-2107. 
Galanti, B., Gulitsky, G., Kholmyansky, M., Tsinober, A. \& Yorish, S. 2003 Velocity derivatives in turbulent flow in an atmospheric boundary layer without Taylor hypothesis. In Turbulence and Shear Flow Phenomena (ed. N. Kasagi, J. K. Eaton, R. Friedrich, J. A. C. Humphrey, M. A. Leschziner \& T. Miyauchi), vol. 2, pp. 745-750.

Galanti, B., Gulitsky, G., Kholmyansky, M., Tsinober, A. \& Yorish, S. 2004 Joint statistical properties of fine structure of velocity and passive scalar in high Reynolds number flows. Adv. Turbulence 10, 267-270.

Gulitski, G., Kholmyansky, M., Kinzelbach, W., LÜthi, B., Tsinober, A. \& Yorish, S. $2007 a$ Velocity and temperature derivatives in high-Reynolds-number turbulent flows in the atmospheric surface layer. Part 1 . Facilities, methods and some general results. J. Fluid Mech. 589, 57-81.

Gulitski, G., Kholmyansky, M., Kinzelbach, W., LÜthi, B., Tsinober, A. \& Yorish, S. $2007 \mathrm{~b}$ Velocity and temperature derivatives in high-Reynolds-number turbulent flows in the atmospheric surface layer. Part 3. Temperature and joint statistics of temperature and velocity derivatives. J. Fluid Mech. 589, 103-123.

Gylfason, A., Ayyalasomayajula, S. \& Warhaft, Z. 2004 Intermittency, pressure and acceleration statistics from hot-wire measurements in wind-tunnel turbulence. J. Fluid Mech. 501, 213-229.

HiLl, R. J. 2002 Scaling of acceleration in locally isotropic turbulence. J. Fluid Mech. 452, 361-370.

Hill, R. J. \& Thoroddsen, S. T. 1997 Experimental evaluation of acceleration correlations for locally isotropic turbulence. Phys. Rev. E 55, 1600-1606.

Hill, R. J. \& WilczaK, J. M. 1995 Pressure structure functions and spectra for locally isotropic turbulence. J. Fluid Mech. 296, 247-269.

HuA, B. L. \& KLeIN, P. 1998 An exact criterion for the stirring properties of nearly two-dimensional turbulence. Physica D 113, 98-110.

Jørgensen, J. B., Mann, J., Ott, S., Pecseli, H. L. \& Trulsen, J. 2005 Experimental studies of occupation and transit times in turbulent flows. Phys. Fluids 17 (3), 035111.

Kholmyansky, M. \& TsinOber, A. 2000 On the origins of intermittency in real turbulent flows. In Proceedings of the Symposium on Intermittency in turbulent flows and other dynamical systems held at Isaac Newton Institute, Cambridge, June 21-24, 1999 (ed. J. C. Vassilicos). Isaac Newton Institute for Mathematical Sciences, Preprint NI99017-TRB. Cambridge University Press.

Kholmyansky, M., Tsinober, A. \& Yorish, S. 2000 Geometrical statistics in the atmospheric turbulent flow at $R e_{\lambda}=10^{4}$. Adv. Turbulence 8, 895-898.

Kholmyansky, M., Tsinober, A. \& Yorish, S. 2001 $a$ Velocity derivatives in the atmospheric surface layer at $R e_{\lambda}=10^{4}$. Phys. Fluids 13, 311-314.

Kholmyansky, M., Tsinober, A. \& Yorish, S. $2001 b$ Velocity derivatives in the atmospheric surface layer at $R e_{\lambda}=10^{4}$. Further results. In Proceedings of the Second International Symposium on Turbulence and Shear Flow Phenomena, Stockholm, June, 27-29, 2001 (ed. E. Lindborg, A. Johansson, J. Eaton, J. Humphrey, N. Kasagi, M. Leschziner \& M. Sommerfeld), vol. 1, pp. 109-113.

la Porta, A., Voth, G. A., Crawford, A. M., Alexander, J. \& Bodenschatz, E. 2001 Fluid particle accelerations in fully developed turbulence. Nature 409, 1017-1019.

LeE, C., Yeo, K. \& ChOI, J.-I. 2004 Intermittent nature of acceleration in near wall turbulence. Phys. Rev. Lett. 92, 144502/1-4.

LÜthi, B., Burr, U., Gyr, A., Kinzelbach, W. \& Tsinober, A. 2001 Velocity derivatives in turbulent flow from 3D-PTV measurements. In Proceedings of the Second International Symposium on Turbulence and Shear Flow Phenomena, Stockholm, June, 27-29, 2001 (ed. E. Lindborg, A. Johansson, J. Eaton, J. Humphrey, N. Kasagi, M. Leschziner \& M. Sommerfeld), vol. 2, pp. $123-128$.

LÜThi, B., Tsinober, A. \& KinZelbaCh, W. 2005 Lagrangian measurement of vorticity dynamics in turbulent flow. J. Fluid Mech. 528, 87-118.

Mann, J., Ott, S. \& Andersen, J. S. 1999 Experimental study of relative, turbulent diffusion. Tech. Rep. RISOE-R-1036(EN). RISOE Natl Lab. Roskilde, Denmark.

Mann, J., Ott, S., Pecseli, H. L. \& Trulsen, J. 2005 Turbulent particle flux to a perfectly absorbing surface. J. Fluid Mech. 534, 1-21.

Monin, A. S. \& Yaglom, A. M. 1975 Statistical Fluid Mechanics, vol. 2. MIT Press. 
Mordant, N., Delour, J., Léveque, E., Michel, O., Arnéodo, A. \& Pinton, J.-F. 2003 Lagrangian velocity fluctuations in fully developed turbulence: scaling, intermittency, and dynamics. J. Stat. Phys. 113, 701-717.

Mordant, N., Crawford, A. M. \& Bodenschatz, E. 2004a Experimental Lagrangian acceleration probability density function measurement. Physica D 193, 245-251.

Mordant, N., Crawford, A. M. \& Bodenschatz, E. 2004b Three-dimensional structure of the Lagrangian acceleration in turbulent flows. Phys. Rev. Lett. 93, 214501/1-4.

Mordant, N., LÉvêQue, E. \& Pinton, J.-F. 2004c Experimental and numerical study of the Lagrangian dynamics of high Reynolds turbulence. New J. Phys. 6, 116/1-44.

Obukhov, A. M. \& Yaglom, A. M. 1951 The microstructure of turbulent flow. Prikl. Mat. Mekh. 15, 3-26, english translation in NACA TM 1350, National Advisory Committee for Aeronautics, Washington, DC, June 1953.

OTt, S. \& MANN, J. 2000 An experimental investigation of the relative diffusion of particle pairs in three-dimensional turbulent flow. J. Fluid Mech. 422, 207-223.

Pinsky, M., Khain, A. \& Tsinober, A. 2000 Accelerations in isotropic and homogeneous turbulence and Taylor's hypothesis. Phys. Fluids 12, 3195-3204.

Pope, S. B. 2002 A stochastic Lagrangian model for acceleration in turbulent flows. Phys. Fluids 14, 2360-2375, erratum: Phys. Fluids 15, 269.

Reynolds, A. M., Mordant, N., Crawford, A. M. \& Bodenschatz, E. 2005 On the distribution of Lagrangian accelerations in turbulent flows. New J. Phys. 7, 58/1-9.

Sawford, B. L., Yeung, P. K., Borgas, M. S., Vedula, P., La Porta, A., Crawford, A. M. \& Bodenschatz, E. 2003 Conditional and unconditional acceleration statistics in turbulence. Phys. Fluids 15, 3478-3489.

Shaw, R. A. 2003 Particle-turbulence interactions in atmospheric clouds. Annu. Rev. Fluid Mech. 35, $183-227$.

Shaw, R. A. \& Oncley, S. P. 2001 Acceleration intermittency and enhanced collision kernels in turbulent clouds. Atmos. Res. 59-60, 77-87.

TAYLOR, G. I. 1935 The statistical theory of turbulence. Proc. R. Soc. Lond. A 151, 421-478.

Tennekes, H. 1975 Eulerian and Lagrangian time microscales in isotropic turbulence. J. Fluid Mech. 67, 561-567.

Tsinober, A. 2001 An Informal Introduction to Turbulence. Kluwer.

Tsinober, A., Kit, E. \& Dracos, T. 1992 Experimental investigation of the field of velocity gradients in turbulent flows. J. Fluid Mech. 242, 169-192.

Tsinober, A., Shtilman, L. \& Vaisburd, H. 1997 A study of vortex stretching and enstrophy generation in numerical and laboratory turbulence. Fluid Dyn. Res. 21, 477-494.

Tsinober, A., Vedula, P. \& Yeung, P. K. 2001 Random Taylor hypothesis and the behavior of local and convective accelerations in isotropic turbulence. Phys. Fluids 13, 1974-1984.

Vedula, P. \& Yeung, P. K. 1999 Similarity scaling of acceleration and pressure statistics in numerical simulations of isotropic turbulence. Phys. Fluids 11, 1208-1220.

Voth, G. A., Satyanarayan, K. \& Bodenschatz, E. 1998 Lagrangian acceleration measurements at large Reynolds numbers. Phys. Fluids 10, 2268-2280.

Voth, G. A., La Porta, A., Crawford, A. M., Alexander, J. \& Bodenschatz, E. 2002 Measurement of fluid particle accelerations in fully developed turbulence. J. Fluid Mech. 469, 121-160.

Vukoslavcevic, P., Wallace, J. M. \& Balint, J.-L. 1991 The velocity and vorticity vector fields of a turbulent boundary layer. Part 1 . Simultaneous measurement by hot-wire anemometry. J. Fluid Mech. 228, 25-51.

Yaglom, A. M. 1949 On the field of accelerations in turbulent flow. Dokl. Akad. Nauk SSSR 67, 795-798.

YeUNG, P. K. 1997 One- and two-particle Lagrangian acceleration correlations in numerically simulated homogeneous turbulence. Phys. Fluids 9, 2981-2990.

YeunG, P. K. \& Borgas, M. S. 2004 Relative dispersion in isotropic turbulence. Part 1. Direct numerical simulations and Reynolds-number dependence. J. Fluid Mech. 503, 93-124.

Yeung, P. K. \& PoPe, S. B. 1989 Lagrangian statistics from direct numerical simulations of isotropic turbulence. J. Fluid Mech. 207, 531-586.

Yeung, P. K., Pope, S. B., Lamorgese, A. G. \& Donzis, D. A. 2006 Acceleration and dissipation statistics of numerically simulated isotropic turbulence. Phys. Fluids 18, 065103. 